\title{
ANÁLISE POR INJEÇÃO SEQUENCIAL COM DETECÇÃO POR VOLTAMETRIA DE ONDA QUADRADA PARA DETERMINAÇÃO DE As(III) EM AMOSTRAS DE ÁGUAS USANDO ELETRODO DE OURO
}

\author{
Fernando Henrique do Nascimento e Jorge Cesar Masini* \\ Departamento de Química Fundamental, Instituto de Química, Universidade de São Paulo, Av. Prof. Lineu Prestes 748, 05508-000 \\ São Paulo - SP, Brasil
}

Recebido em 25/07/2017; aceito em 18/09/2017; publicado na web em 8/11/2017

\begin{abstract}
SEQUENTIAL INJECTION ANALYSIS WITH SQUARE WAVE VOLTAMMETRY DETECTION AT GOLD ELECTRODE FOR DETERMINATION OF As(III) IN WATER SAMPLES. A sequential injection method with square wave anodic stripping voltammetry (SI-SWASV) using screen printed and crystalline gold electrode was developed for determination of As(III). The carrier solution used for electrode conditioning and stripping medium was composed of $0.050 \mathrm{~mol} \mathrm{~L}^{-1} \mathrm{HCl}$, whereas the supporting electrode for samples (deposition medium) was $0.50 \mathrm{~mol} \mathrm{~L}^{-1} \mathrm{HCl}$. The limits of detection and quantification were 20 and $70 \mathrm{nmol} \mathrm{L}^{-1}\left(1.5\right.$ and $\left.5.2 \mu \mathrm{g} \mathrm{L} \mathrm{L}^{-1}\right)$, respectively. This limit of quantification is almost two times lower than the maximum concentration of As(III) allowed in waters by World Health Organization $\left(10 \mu \mathrm{g} \mathrm{L}^{-1}\right)$. The sampling throughput was 20 analyses per hour using a sample volume of $1500 \mu \mathrm{L}$, pumped through the flow cell at the flow rate of $15 \mu \mathrm{L} \mathrm{s}^{-1}$. Screen printed gold electrodes were not suitable for work with $0.50 \mathrm{~mol} \mathrm{~L}^{-1}$ $\mathrm{HCl}$ because the results were strongly dependent on the lot of sensors. Crystalline gold electrodes provided reproducible results and the method was applied for determination of As(III) in river water samples. The accuracy was evaluated by recovery studies and analyses by Graphite Furnace Atomic Absorption Spectrometry, providing recoveries between 95 and 108\%.
\end{abstract}

Keywords: sequential injection analysis; square wave voltammetry; gold electrodes; arsenic; waters.

\section{INTRODUÇÃO}

Em ambientes aquáticos o arsênio pode ocorrer nos estados de oxidação +3 e +5 nas formas inorgânicas de $\mathrm{AsO}_{3}{ }^{3-}$ e $\mathrm{AsO}_{4}{ }^{3-}$, ou na forma de compostos orgânicos. ${ }^{1}$ Compostos de arsênio têm recebido atenção especial devido aos riscos de contaminação de ambientes aquáticos, sendo que seus compostos trivalentes são cerca de 60 vezes mais tóxicos do que os pentavalentes. Além disso, os compostos inorgânicos são mais tóxicos do que os orgânicos, sendo que todos eles promovem efeitos carcinogênicos. A principal rota de intoxicação humana é o consumo de água contaminada, especialmente de água subterrânea, resultando em doenças de pele, bexiga, câncer de pulmão, problemas reprodutivos e de desenvolvimento, doenças cardiovasculares, etc. Em função dessa problemática, As é considerado um poluente prioritário pela Agência de Proteção Ambiental dos Estados Unidos (US-EPA), de modo que sua monitoração em águas é essencial para evitar problemas de saúde pública, como ocorridos em Bangladesh. ${ }^{1,2}$ Para determinação de As(III), as técnicas mais comumente utilizadas são a espectrometria de massas com plasma indutivamente acoplado (ICP-MS), a espectrometria de fluorescência atômica e a espectrometria de absorção atômica. A atomização pode ser realizada em forno de grafite ou por geração de hidretos, sendo essas duas últimas capazes de promover a separação do analito da matriz, reduzindo o efeito de vários interferentes. ${ }^{3}$ Essas técnicas podem ser acopladas a cromatografia líquida de alta eficiência para fins de especiação. ${ }^{3}$ Apesar de fornecerem alta sensibilidade e baixos limites de detecção, tais técnicas têm alto custo de instrumentação e manutenção, além de não poderem ser usadas para determinações em campo.

Os métodos eletroanalíticos são conhecidos pelo uso de equipamentos de baixo custo de instrumentação e elevada portabilidade. São seletivos a As(III) e apresentam sensibilidade e detectabilidade similares àquelas fornecidas pelas técnicas de absorção ou emissão

*e-mail: jcmasini@iq.usp.br atômica. ${ }^{4}$ A voltametria de redissolução pode ser usada na forma anódica e catódica, usando-se diferentes tipos de eletrodo de trabalho, como platina, ouro, substratos de carbono e diamante dopado com boro. ${ }^{2,4-8} \mathrm{O}$ ouro, dentre os diferentes substratos disponíveis, é o que promove maior sensibilidade para a determinação do As, além de possuir um maior sobrepotencial de hidrogênio do que a Pt. ${ }^{9}$

Medidas voltamétricas ou amperométricas realizadas em fluxo melhoram a repetitividade dos resultados, uma vez que as condições de transporte de massa do seio da solução para a superfície do eletrodo são bastante reprodutíveis nas condições de vazão e diâmetro de tubos tipicamente utilizados em análise por injeção em fluxo (FIA) ou injeção sequencial (SIA). ${ }^{10-12}$ Determinações voltamétricas e amperométricas de As(III) em sistemas de fluxo já foram descritas, ${ }^{13-16}$ mas ainda foram pouco estudadas em sistemas SIA. ${ }^{14}$

Duangthong e colaboradore ${ }^{13}$ propuseram a determinação de As(III) e As(IV) por voltametria de pulso diferencial usando um eletrodo de filme de ouro depositado sobre carbono vítreo. Apesar dos autores definirem o método como sendo de análise por injeção em fluxo, trata-se na realidade de um método de análise em fluxo contínuo. Após o preparo do eletrodo de trabalho, a amostra foi continuamente bombeada na cela de fluxo enquanto o potencial de deposição era aplicado para redução de As(III) a As ${ }^{0}$. Após um tempo adequado, a reoxidação do $\mathrm{As}^{0}$ depositado no eletrodo era feita por voltametria de pulso diferencial.

Dois artigos envolvendo FIA descrevem métodos indiretos explorando a reação entre espécies de As e $\mathrm{I}_{2} \cdot{ }^{15,16}$ No primeiro caso um sensor impresso de grafite com a configuração disco-anel foi usado em uma cela do tipo wall-jet. Nesse caso o $\mathrm{I}_{2}$ foi gerado eletroquimicamente a partir de uma solução de $\mathrm{I}^{-}$no disco. O sinal analítico baseou-se na diminuição da corrente de redução do $\mathrm{I}_{2}$ no anel devido à reação do $\mathrm{I}_{2}$ com $\mathrm{As}(\mathrm{III})$ em solução. ${ }^{16}$ Lolic e colaboradores ${ }^{15}$ propuseram um método com difusão gasosa no qual As(III) e As(IV) foram reduzidos a arsina por $\mathrm{NaBH}_{4}$. A arsina difundida através de uma membrana de PTFE reagiu na solução coletora composta por $\mathrm{I} / \mathrm{I}_{2}$ fluindo continuamente pela cela de fluxo contendo um eletrodo 
de trabalho de Pt. Nesse caso o pico de redução do sistema $\mathrm{I}_{2} / \mathrm{I}^{-} \mathrm{em}$ $0,10 \mathrm{~V}$ (vs $\mathrm{Ag} / \mathrm{AgCl}, \mathrm{KCl} 3 \mathrm{~mol} \mathrm{~L}^{-1}$ ) diminui com o aumento da concentração de As(III).

Punrat e colaboradores ${ }^{14}$ descreveram um método automatizado por SIA para determinação de As(III) e As total por voltametria de redissolução usando um eletrodo de ouro depositado sobre um sensor impresso de grafite. O eletrodo de trabalho foi considerado um eletrodo de longa duração, uma vez que foi utilizado durante três dias trabalho, realizando mais de trezentas análises e mantendo um desvio padrão relativo de $2,7 \%$. O método baseou-se na injeção de $1200 \mu \mathrm{L}$ de amostra (em $\mathrm{HCl} 1,0 \mathrm{~mol} \mathrm{~L}^{-1}$ ) na cela de fluxo, aplicando-se um potencial de deposição de $-0,50 \mathrm{~V}$ vs. $\mathrm{Ag} / \mathrm{AgCl}$, seguindo-se a etapa de redissolução com varredura linear de potencial até $0,40 \mathrm{~V}$. Duas etapas de limpeza do eletrodo foram usadas, injetando-se em cada uma, $2500 \mu \mathrm{L}$ de $\mathrm{HCl} 1 \mathrm{~mol} \mathrm{~L}^{-1}$ na cela de fluxo, mantendo-se o potencial do eletrodo de trabalho em $0,40 \mathrm{~V}$ vs $\mathrm{Ag} / \mathrm{AgCl}$.

O presente trabalho apresenta o desenvolvimento de método de análise por injeção sequencial com detecção por voltametria de redissolução anódica por onda quadrada (SI-SWASV) para determinação de As(III) em amostras de águas usando eletrodos comerciais de ouro cristalino e sensores impressos de ouro. $\mathrm{O}$ uso desses eletrodos evita a necessidade de preparo de eletrodos de filme de Au, que envolve o consumo de soluções de $\mathrm{Au}(\mathrm{III})$. Serão discutidas vantagens e desvantagens do emprego de sensores impressos de ouro comparativamente a eletrodos de ouro cristalino usando um sistema SIA em sua configuração mecânica mais simples, constituída por uma bomba de pistão, uma válvula seletora e o detector.

\section{PARTE EXPERIMENTAL}

\section{Aparelhos e reagentes}

As soluções de ácido clorídrico utilizadas como eletrólito suporte ou solução transportadora foram preparadas com reagente de alta pureza, Surprapur ${ }^{\circledR}$ da Merck (Rio de Janeiro, Brasil). Todos os outros reagentes foram de grau analítico, sendo usados sem purificação adicional. As soluções foram preparadas com água desionizada até resistividade $>18 \mathrm{M} \Omega \mathrm{cm}$ em um sistema Simplicity 185 da Millipore (Billerica, MA, EUA).

A manipulação das soluções foi feita por meio de um sistema de injeção sequencial montado no laboratório, esquematizado na Figura 1. As soluções foram conduzidas por uma bomba de seringa (BS) Cavro, SP (Cavro Scientific Instruments, Sunnyvale, CA) com capacidade de 5,0 $\mathrm{mL}$ e uma válvula rotatória (VR) de 8 portas (Valco Instrument Co., Houston, TX). A bomba de seringa e a válvula rotatória foram conectadas através de uma válvula de três vias, VS (posições in e out), pela bobina coletora, BC, feita com tubo de Teflon ${ }^{\circledR}$ de 3 m de comprimento com 1,0 mm de diâmetro interno e, portanto, 2,36 mL de volume interno. O volume interno deve ser desta magnitude para evitar a contaminação da solução transportadora no interior da seringa com a solução da amostra. Conexões das portas da válvula rotatória com as soluções e a célula de detecção foram feitas com tubos de Teflon com diâmetro interno de $0,5 \mathrm{~mm}$ e conexões de Teflon (Upchurch, Oak Harbor, WA). Os controles da bomba e da válvula foram efetuados com o software FIAlab 5.0.

Medidas voltamétricas foram realizadas em um potenciostato PalmSens (PalmSens Instrument BV, Houten, Holanda) usando o software PSTrace 2.4 para controle de instrumentos e aquisição de dados. Uma das celas de fluxo foi montada usando o sensor impresso da Universidade de Florença, composto por um eletrodo de trabalho de ouro (área de 7,06 $\mathrm{mm}^{2}$ e $450 \mathrm{~mm}$ de espessura), um pseudo referência de $\mathrm{Ag}$ e um contra eletrodo de grafite. ${ }^{17,18}$ Adicionalmente, medidas foram feitas em cela de fluxo de camada delgada da Bioanalytical Systems (West Lafayette, IN, EUA) usando eletrodo duplo de ouro cristalino ( $3 \mathrm{~mm}$ de diâmetro), sendo um desses eletrodos usado como eletrodo auxiliar. Como referência foi usado um eletrodo de $\mathrm{Ag} / \mathrm{AgCl}\left(\mathrm{NaCl} 3 \mathrm{~mol} \mathrm{~L}^{-1}\right)$ modelo RE-6 da Bioanalytical System.

\section{Condicionamento dos eletrodos}

A ativação do eletrodo impresso de ouro (SPGE) foi feita diretamente na cela de fluxo, baseando-se no procedimento de Bernalte et al. ${ }^{19} \mathrm{em}$ meio de $\mathrm{HCl}$ 0,050 mol L-1 (solução transportadora usada para preenchimento da célula eletroquímica). Para isso, a seringa do sistema SIA foi preenchida com $2000 \mu \mathrm{L}$ da solução transportadora e esse volume foi bombeado para a célula eletroquímica a uma vazão de $200 \mu \mathrm{L} \mathrm{s}^{-1}$. Em seguida, com o fluxo parado, o potenciostato aplicou um potencial de $0,7 \mathrm{~V}$ por $15 \mathrm{~s}$, seguido de 10 ciclos de voltametria cíclica na faixa de potencial de 0 a $0,7 \mathrm{~V}$ a $0,050 \mathrm{~V} \mathrm{~s}^{-1} .^{20,21}$

Para o eletrodo de ouro cristalino, inicialmente foi feito o polimento com suspensão de diamante com partículas de $0,25 \mu \mathrm{m}$ de diâmetro sobre pano autoadesivo de polimento metalográfico (Arotec, São Paulo, SP) por 5 minutos, seguido por tratamento em banho de ultrassom por mais 5 minutos. O eletrodo foi, então, condicionado em meio de $\mathrm{HCl} 0,05 \mathrm{~mol} \mathrm{~L}^{-1}$ seguindo procedimento similar ao descrito no parágrafo anterior.

\section{Procedimento de análise}

A determinação voltamétrica de As(III) automatizada por SIA foi baseada no instrumento esquematizado na Figura 1 . O procedimento é constituído em 15 etapas, conforme descrito na Tabela 1.

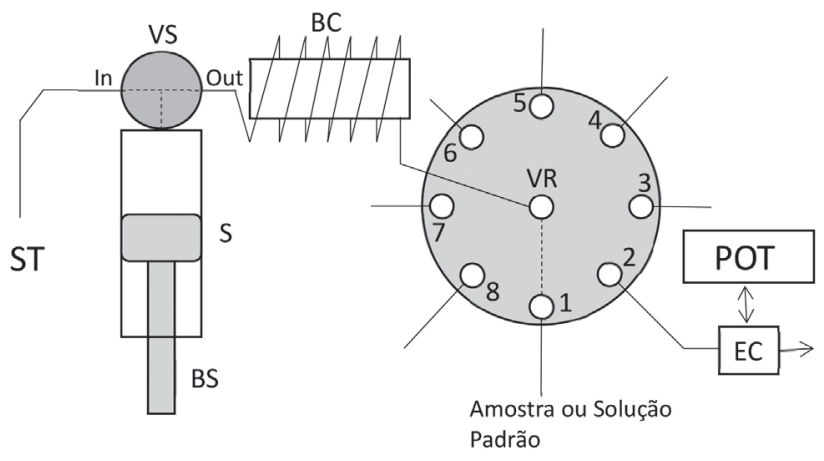

Figura 1. Sistema de injeção sequencial para a determinação de As(III) com detecção por voltametria de onda quadrada. ST - Solução Transportadora, $\mathrm{HCl}$ 0,05 mol L ${ }^{-1}$, VS - Válvula da Seringa (posições In e Out), S - Seringa, BS - Bomba de Seringa, BC - Bobina Coletora $(3 \mathrm{~m}$ de tubulação de tubo de Teflon com 1,0 mm d.i.), VR - Válvula Rotatória; POT - Potenciostato; CE - Cela Eletroquímica

Apesar do condicionamento inicial dos eletrodos, a repetitividade dos experimentos é melhorada quando uma etapa inicial de condicionamento e limpeza eletroquímica é realizada aplicando-se um potencial de $0,40 \mathrm{~V}$ ao eletrodo de $\mathrm{Au}$ frente à referência (etapas 1 a 6, Tabela 1) antes de iniciar a deposição (etapa 9). Em seguida o volume de amostra é aspirado para a bobina coletora (BC), enquanto o potenciostato inicia a aplicação do potencial de deposição (etapas 7 a 9). Após aspiração da amostra em BC, a direção do fluxo é revertida e a solução da amostra é injetada na cela eletroquímica à vazão de $15 \mu \mathrm{L} \mathrm{s}^{-1}$ através da porta 2 da VR (etapas 10 e 11). Após a troca de meio para a solução transportadora (etapa 12) o fluxo é parado por $10 \mathrm{~s}$ (etapa 13) para realização da varredura de potencial e redissolução do $\mathrm{As}^{0}$. Para a obtenção de resultados reprodutíveis, o potencial de 
Tabela 1. Sequência de operações do sistema SIA para determinação de As(III)

\begin{tabular}{lcccc}
\hline Etapa & Componente & Comando & Parâmetro/posição & Comentário \\
\hline 1 & POT & Aplica & $0,4 \mathrm{~V}$ por $70 \mathrm{~s}$ & Potencial de limpeza/condicionamento \\
2 & VS & Comuta & In & \\
3 & BS & Aspira & $3000 \mu \mathrm{L} \mathrm{a} 200 \mu \mathrm{L} \mathrm{s}^{-1}$ & Enchendo a seringa com solução transportadora \\
4 & VS & Comuta & Out & \\
5 & RV & Comuta & Porta 2 & Condicionamento eletroquímico e limpeza de cela \\
6 & BS & Dispensa & $1000 \mu \mathrm{L} \mathrm{a} 100 \mu \mathrm{L} \mathrm{s}^{-1}$ & Aspirando a amostra para BC \\
7 & VR & Comuta & Porta 1 & Aplicando potencial de deposição \\
8 & BS & Aspira & $1500 \mu \mathrm{L} \mathrm{a} 50 \mu \mathrm{L} \mathrm{s}{ }^{-1}$ & Injetando a amostra na cela eletroquímica \\
9 & POT & Aplica & $-0,5 \mathrm{~V}$ por $120 \mathrm{~s}$ & Trocando o meio de deposição para redissolução \\
10 & VR & Comuta & Porta 2 & Parada de fluxo para início da redissolução \\
11 & BS & Dispensa & $1500 \mu \mathrm{L} \mathrm{a} 15 \mu \mathrm{L} \mathrm{s}{ }^{-1}$ & Etapa de redissolução sob parada de fluxo \\
12 & BS & Dispensa & $500 \mu \mathrm{L} \mathrm{a} 50 \mu \mathrm{L} \mathrm{s}$ &
\end{tabular}

deposição deve ser aplicado desde antes do início do bombeamento da zona de amostra na cela de fluxo, e mantido aplicado até a parada de fluxo após a troca de meio. Finalmente a solução excedente no interior da seringa é esvaziada para limpeza da cela eletroquímica (etapa 14).

\section{Amostras}

Amostras de água foram coletadas nos rios Ribeirão do Meio e Lençóis no município de Lençóis, BA. As amostras foram filtradas em membrana de acetato de celulose de $0,45 \mu \mathrm{m}$ de porosidade, acondicionados em $\mathrm{HCl} 0,50 \mathrm{~mol} \mathrm{~L}^{-1}$ e armazenadas em refrigerador $\left(4^{\circ} \mathrm{C}\right)$ até o momento da análise. A exatidão dos resultados foi verificada por estudos de adição e recuperação, adicionando o analito de modo a obter uma concentração de $10 \mu \mathrm{g} \mathrm{L}^{-1}$ considerando a ausência de As na amostra. Para checar a exatidão do método proposto as amostras foram analisadas por espectrometria de absorção atômica em forno de grafite em um espectrômetro ZEEnit (AnalyticJena, Jena, Alemanha) pelo Grupo de Análises e Pesquisa em Espectrometria (GAPE) do Instituto de Química da Universidade de São Paulo, segundo método descrito por Correia e Oliveira. ${ }^{22}$

\section{RESULTADOS E DISCUSSÃO}

\section{Efeito da concentração de $\mathrm{HCl}$}

O desenvolvimento do método SIA-SWASV foi baseado no trabalho de Punrat et al. ${ }^{14}$ com um sensor impresso de carbono sobre o qual um filme de ouro foi depositado eletroquimicamente em meio de $\mathrm{HCl}$ 1,0 mol L-1 . Ácido clorídrico é o eletrólito suporte mais comum, em concentrações que podem variar desde 0,1 a $7 \mathrm{~mol} \mathrm{~L}^{-1} .{ }^{7}$ Apesar do uso frequente de $\mathrm{HCl}$ como eletrólito, os íons cloreto adsorvem fortemente no ouro, afetando a carga do eletrodo, podendo assim afetar a interação com o As(III). ${ }^{23}$ Além disso, altas concentrações de $\mathrm{HCl}$ podem causar a dissolução da superfície do eletrodo do ouro pela complexação com íons cloreto $\left(\mathrm{AuCl}_{4}^{-}\right)$, reduzindo a área superficial e acarretando em perda de sensibilidade das medidas com o tempo. ${ }^{9}$

Assim, inicialmente foi avaliado o efeito da concentração de $\mathrm{HCl}$ como eletrólito suporte da amostra (ES), como solução transportadora e meio de redissolução (STR), e como solução de ativação (SA). Para isto, foram feitos experimentos com soluções de As(III) 1,0 $\mu \mathrm{mol} \mathrm{L} \mathrm{L}^{-1}$ e diferentes combinações de concentrações de $\mathrm{HCl}$ em ES, STR e SA (Figura 2). O procedimento de troca de meio ${ }^{20,21}$ foi adotado para aumentar a eficiência da reoxidação do $\mathrm{As}^{0}$, supondo a formação de cloro-complexos $\left(\mathrm{AsCl}_{\mathrm{n}}{ }^{3-\mathrm{n}}\right)$.

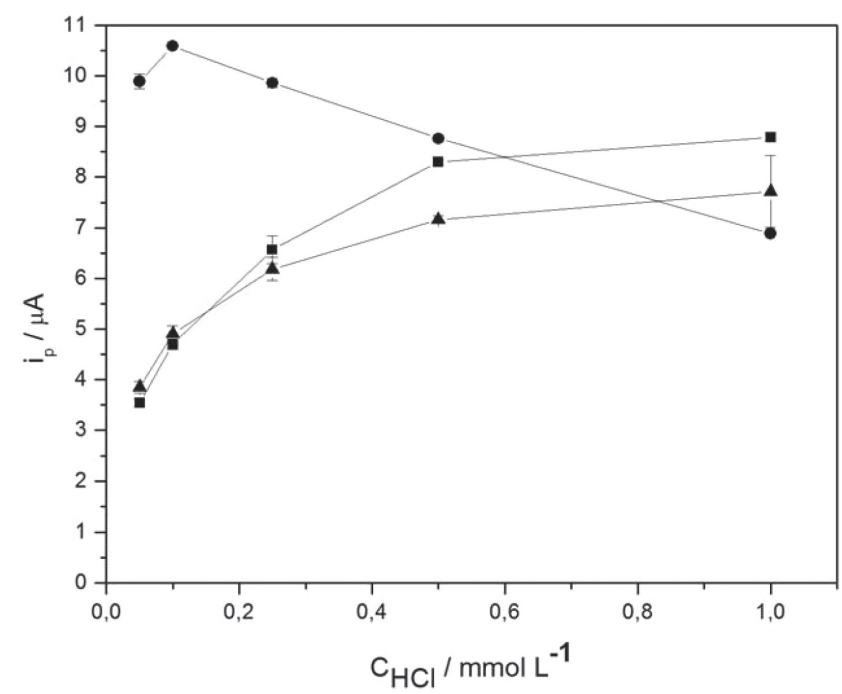

Figura 2. Efeito da concentração de $\mathrm{HCl}$ como ES nas correntes de pico,

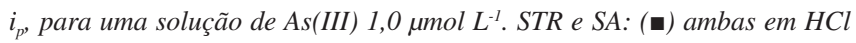
$0,050 \mathrm{~mol} \mathrm{~L}^{-1}$, ( $\mathbf{\Delta}$ ) 0,050 e 1,0 mol L-1, respectivamente e $(\bullet)$ ambas em $\mathrm{HCl}$

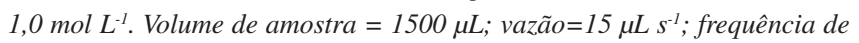
pulsos $=100 \mathrm{~Hz}$

As correntes de pico $\left(i_{p}\right)$ aumentaram sistematicamente com o aumento da concentração de $\mathrm{HCl}$ no ES quando as soluções de STR e SA foram ambas $0,050 \mathrm{~mol} \mathrm{~L}^{-1}$ (Figura 2, a) ou 0,050 e 1,0 $\mathrm{mol} \mathrm{L}^{-1}$ (Figura 2, $\boldsymbol{\Delta}$ ), respectivamente. Os maiores valores de $i_{p}$ foram obtidos usando-se $\mathrm{HCl}$ 1,0 mol L-1 como STR e SA, bem como $\mathrm{HCl}$ $0,10 \mathrm{~mol} \mathrm{~L}^{-1} \mathrm{em} \mathrm{ES}$, sugerindo que essas seriam as concentrações ótimas (Figura 2, $\bullet$ ), de modo consistente com o trabalho de Punrat et al. ${ }^{14}$ No entanto, quando $\mathrm{HCl} 1,0 \mathrm{~mol} \mathrm{~L}^{-1}$ foi usado como STR e SA houve diminuição de $\mathrm{i}_{\mathrm{p}}$ para concentrações de $\mathrm{HCl}$ maiores do 
que $0,10 \mathrm{~mol} \mathrm{~L}^{-1}$ em ES (Figura 2, •). Usando-se $\mathrm{HCl}$ 0,050 $\mathrm{mol} \mathrm{L}^{-1}$ como STR e SA, e $\mathrm{HCl} 0,50 \mathrm{~mol} \mathrm{~L}^{-1}$ como ES, os valores de $\mathrm{i}_{\mathrm{p}}$ sofreram uma diminuição de $22 \%$ em relação ao máximo obtido. Por outro lado, esta condição permitiu o trabalho com soluções de $\mathrm{HCl}$ menos concentradas, diminuindo o consumo de reagentes e a geração de resíduos, além de contribuir com uma maior vida útil da instrumentação, especialmente de componentes metálicos do pistão e da válvula rotatória do sistema SIA. Adicionalmente, conferiu maior robustez ao método, pois minimizou a perda da sensibilidade das medidas com o tempo. ${ }^{9}$ Como discutido anteriormente, essa perda de sensibilidade pode ser causada pelas altas concentrações de $\mathrm{Cl}^{-}$.

As condições descritas, entretanto, não foram sempre apropriadas para os sensores impressos de ouro, que dependendo do lote de fabricação podiam ser dissolvidos na etapa de condicionamento em $\mathrm{HCl}$ com concentrações $>0,50 \mathrm{~mol} \mathrm{~L}^{-1}$. Essa observação é consistente com a proposta de Punrat et al., ${ }^{14}$ que prepararam os eletrodos por deposição eletroquímica de Au sobre um sensor impresso de carbono em meio de $\mathrm{HCl}$ 1,0 mol L-1, denominando tais sensores como de longa duração. Em função dessa limitação dos sensores impressos comerciais, a sequência do trabalho foi realizada com o eletrodo cristalino de ouro.

\section{Efeito da frequência de pulso, vazão e volume de amostra}

O efeito da frequência de pulso de onda quadrada foi avaliado mantendo-se constantes o volume de amostra em $1000 \mu \mathrm{L}$ e a vazão em $15 \mu \mathrm{L} \mathrm{s}^{-1}$ (Figura 3). O aumento da frequência aumentou sistematicamente os valores de $i_{p}$, de modo consistente com os resultados descritos por Giacomino et al. ${ }^{9}$ Houve ganho de 7 vezes no sinal com o aumento da frequência de 25 para $100 \mathrm{~Hz}$, sendo a frequência de $100 \mathrm{~Hz}$ adotada para o método.

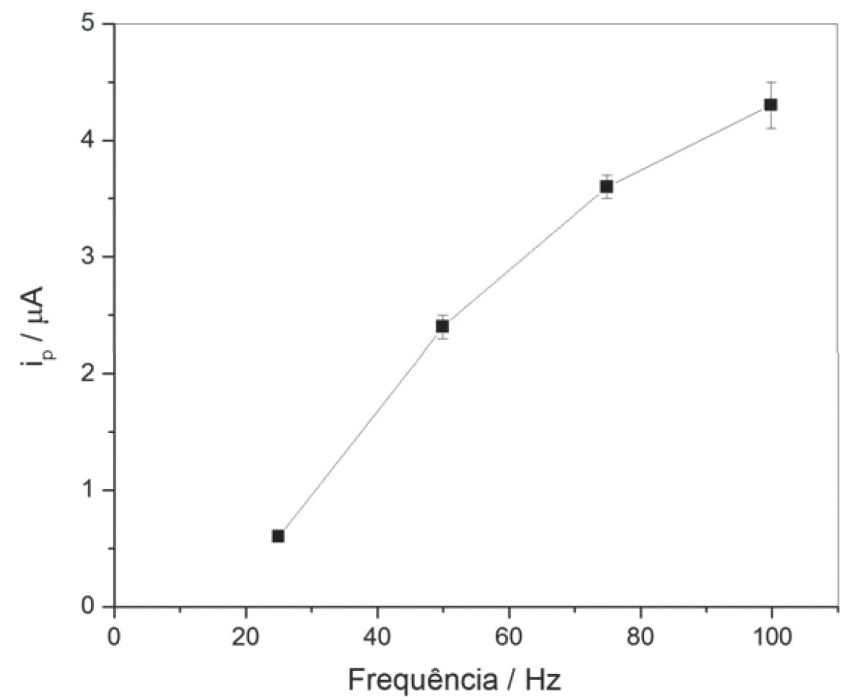

Figura 3. Variação da corrente de pico $\left(i_{p}\right)$ para diferentes frequências de pulsos de onda quadrada para medidas de uma solução de As(III) 1,0 $\mu \mathrm{mol} \mathrm{L}$ L $^{-1}$,

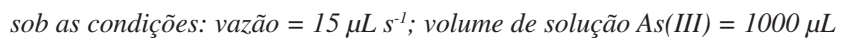

O efeito da vazão foi estudado numa faixa entre 5 e $100 \mu \mathrm{L} \mathrm{s}^{-1}$, com volume de amostra $(1000 \mu \mathrm{L})$ e frequência de pulsos $(100 \mathrm{~Hz})$ fixos (Figura 4). O aumento da vazão de 5 a $100 \mu \mathrm{L} \mathrm{s}^{-1}$ diminuiu em cerca de $80 \%$ o valor de $i_{p}$. Com isso, concluiu-se que o aumento da eficiência do transporte de massa em alta vazão não compensou a diminuição do tempo de deposição para um mesmo volume de amostra.

Quanto maior o volume de amostra usado, maior foi o tempo de deposição necessário para que este passasse pela célula de fluxo durante a etapa de deposição. De modo similar, maiores volumes de

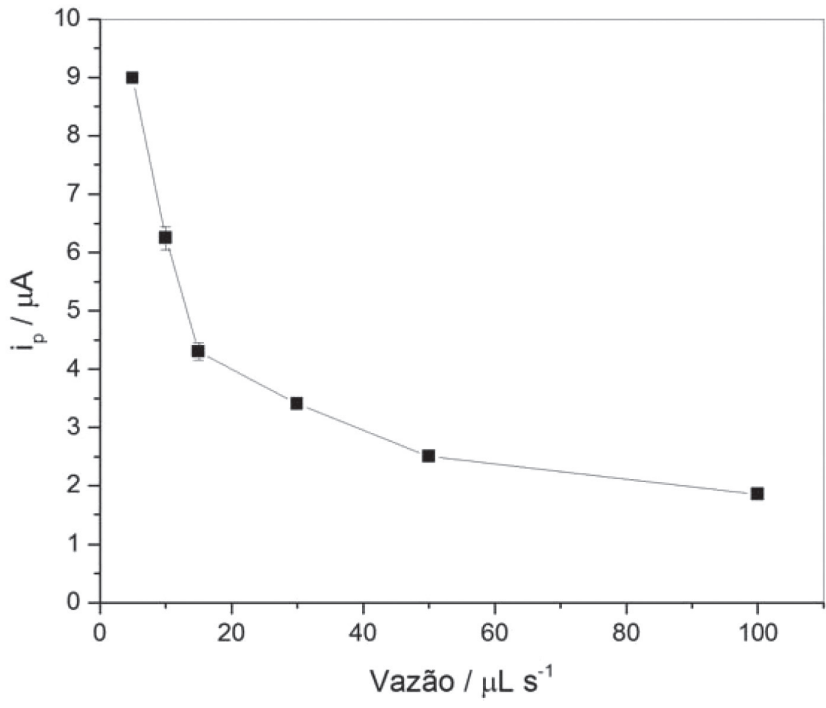

Figura 4. Variação de $i_{p}$ para medidas de uma solução de As(III) 1,0 $\mu \mathrm{mol} \mathrm{L}^{-1}$ sob as condiçôes: tempo de condicionamento $=70 \mathrm{~s}$; tempos de deposição $=210,110,80,45,30$ e 20 s (correspondentes às vazões de 5, 10, 15, 30, 50 e $100 \mu \mathrm{L} \mathrm{s}^{-1}$, respectivamente); volume de amostra $=1000 \mu \mathrm{L}$; e frequência de pulsos $=100 \mathrm{~Hz}$

amostra demandam mais tempo de aspiração para a bobina coletora, mantida a mesma vazão em todos os experimentos. Com isso, o tempo de condicionamento também foi variado nesse caso para garantir que o potencial de deposição fosse aplicado durante todo o período de passagem da zona de amostra pelo detector. Maiores volumes de amostra geraram maiores valores de $\mathrm{i}_{\mathrm{p}}$, de modo consistente com os resultados de Punrat et al..$^{14}$ e Laschi et al. ${ }^{18}$ sendo que o sinal aumentou cerca de 5 vezes com o aumento de volume de 250 para $1500 \mu \mathrm{L}$ (Figura 5).

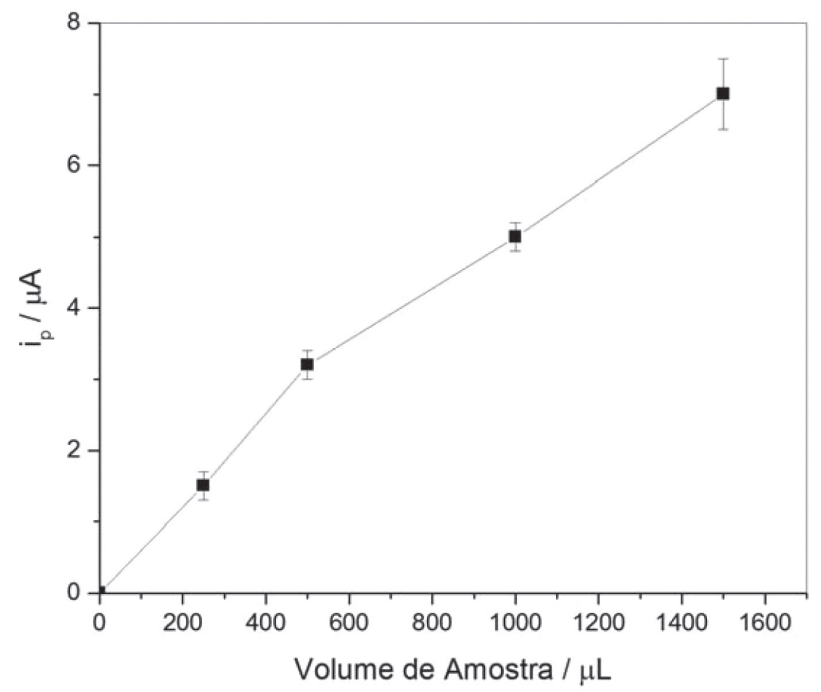

Figura 5. Variação de $i_{p}$ para medidas de uma solução de As(III) 1,0 $\mu$ mol L $L^{-1}$ sob as condições: tempos de condicionamento =60, 65, 70 e $85 \mathrm{~s}$; tempos de deposição $=37,55,87$ e 120 s (correspondentes aos volumes de amostra de 250, 500, 1000 e $1500 \mu \mathrm{L}$, respectivamente); vazão $=15 \mu \mathrm{L} \mathrm{s}^{-1}$ e frequência de pulsos $=100 \mathrm{~Hz}$

Com base nestes resultados, as melhores condições seriam a adoção da vazão de $5 \mu \mathrm{L} \mathrm{s}^{-1}$ e o volume de amostra de $1500 \mu \mathrm{L}$. Todavia, para um volume de amostra de $1500 \mu \mathrm{L}$, um aumento da vazão de 5 para $15 \mu \mathrm{L} \mathrm{s}^{-1}$ implicou em um aumento da frequência de amostragem 
de 10 para 20 determinações por hora, com uma diminuição de apenas $19 \%$ de $i_{p}$. Assim, para uma situação de compromisso entre sensibilidade e frequência de amostragem, a caracterização das figuras de mérito do método foi realizada adotando um volume de amostra de $1500 \mu \mathrm{L}$, injetados a $15 \mu \mathrm{L} \mathrm{s}^{-1}$, usando-se $100 \mathrm{~Hz}$ como frequência de aplicação de pulso na etapa de redissolução (altura de pulso de $30 \mathrm{mV}$ e incremento de potencial de $3 \mathrm{mV}$ ).

\section{Figuras de mérito}

A faixa de resposta linear foi observada para o intervalo de concentrações entre 0,10 e $1,0 \mu \mathrm{mol} \mathrm{L}^{-1}(\mathrm{r}=0,997)$, sendo as soluções de $\mathrm{As}(\mathrm{III})$ preparadas em $\mathrm{HCl} 0,50 \mathrm{~mol} \mathrm{~L}^{-1}$ como eletrólito suporte (Figura 6).

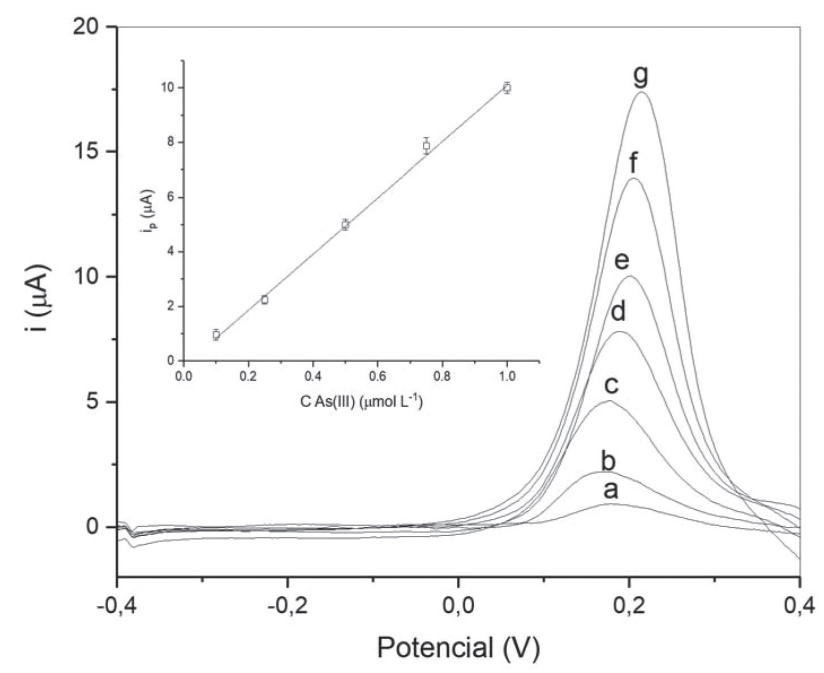

Figura 6. Voltamogramas (subtraídos do branco) obtidos para soluções (a) 0,10, (b) 0,25, (c) 0,50, (d) 0,75, (e) 1,0, (f) 2,5 e (g) 5,0 umol L $L^{-1} d e$ As(III), que resultaram na curva analítica, $i_{p}=(10,3 \pm 0,2) C_{A s(I I I)}-(0,2 \pm 0,1)$, obtida em condições de vazão e volume de amostra de $15 \mu \mathrm{L} \mathrm{s} s^{-1}$ e $1500 \mu \mathrm{L}$, respectivamente. Frequência de pulsos $=100 \mathrm{~Hz}$. Eletrólito suporte e branco: $\mathrm{HCl} 0,50 \mathrm{~mol} \mathrm{~L}^{-1}$

Os limites de detecção (LOD) e quantificação (LOQ) foram calculados em função do desvio padrão das 10 medidas do branco

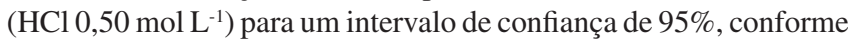
as equações 1 e 2 :

$$
\begin{aligned}
& \mathrm{LOD}=\frac{3 \sigma_{B}}{\mathrm{~m}} \\
& \mathrm{LOQ}=\frac{10 \sigma_{\mathrm{B}}}{\mathrm{m}}
\end{aligned}
$$

em que $\sigma_{\mathrm{B}}$ é o desvio padrão do branco e m é o coeficiente angular da curva analítica.

Os valores de LOD e LOQ foram 0,02 e 0,07 $\mu \mathrm{mol} \mathrm{L}^{-1}$ ( ou 1,5 e $\left.5,2 \mu \mathrm{g} \mathrm{L}^{-1}\right)$, respectivamente. O limite de quantificação foi cerca de 2 vezes menor que a concentração permitida pela organização mundial de saúde (OMS) para As(III) em água potável $\left(10 \mu \mathrm{g} \mathrm{L}^{-1}\right)$. O limite de detecção foi menor do que o obtido por Laschi et al., ${ }^{18} \mathrm{de} 2,5 \mu \mathrm{g} \mathrm{L}^{-1}$, usando o método SWASV em modo estático com tempo de deposição de $60 \mathrm{~s}$, volume de amostra de $2 \mathrm{~mL}$ e sensores impressos de ouro. A maior detectabilidade do método aqui proposto pode ser explicada pela melhor eficiência no transporte de massa obtida nas condições de fluxo usadas na etapa de deposição de $\mathrm{As}^{0}$.
Comparado com outros métodos em fluxo, o LOD do método aqui proposto é menor do que aqueles obtidos por métodos amperométricos indiretos baseados na reação de $\mathrm{As}(\mathrm{III})$ com $\mathrm{I}_{2}$ gerado eletroquimicamente no eletrodo impresso disco-anel $\left(4.0 \mu \mathrm{mol} \mathrm{L}^{-1}\right)^{15}$ ou na reação de arsina com $\mathrm{I}_{2}\left(5.0 \mu \mathrm{mol} \mathrm{L} \mathrm{L}^{-1}\right) .{ }^{16}$ No método descrito por Punrat e colaboradores, ${ }^{14}$ o LOD é significativamente menor $\left(0,03 \mu \mathrm{g} \mathrm{L}^{-1}\right)$, o que pode ser explicado pela forma de preparo do eletrodo de trabalho por eletrodeposição a partir de uma solução de $\mathrm{Au}(\mathrm{III})$ sobre um sensor impresso de grafite. Nesse caso, imagens de microscopia eletrônica de varredura mostraram que o depósito não é de um filme uniforme, mas sim de partículas com diâmetro médio de $570 \pm 83 \mathrm{~nm}$, conferindo uma área efetiva de eletrodo significativamente maior do que a do eletrodo cristalino polido. Por outro lado, como mencionado anteriormente, o método aqui proposto permite monitorar as concentrações de As nos níveis estabelecidos pela OMS, usando condições de trabalho mais simples, sem a necessidade de realizar a eletrodeposição de Au a partir de soluções de Au(III), que apresentam alto custo. Uma possível explicação para isso é uso da voltametria de onda quadrada, na qual a discriminação da corrente faradaica em relação à capacitiva é muito mais eficiente do que na varredura linear usada por Punrat e colaboradores.

A precisão foi avaliada por estudos de repetitividade das correntes de pico obtidos para soluções de As(III) nos níveis de concentração de $0,25,0,50$ e $0,75 \mu \mathrm{mol} \mathrm{L}^{-1}$, em triplicata. Os respectivos desvios padrão relativos para as medidas dessas soluções foram 7,9, 2,8 e 0,7\%.

\section{Interferências}

Foram avaliadas as possíveis interferências da presença de outras espécies iônicas nos valores de $\mathrm{i}_{\mathrm{p}}$ de uma solução de As(III) $1,0 \mu \mathrm{mol} \mathrm{L} \mathrm{L}^{-1}$ na ausência e presença das espécies potencialmente interferentes (Tabela 2).

Tabela 2. Influência de possíveis espécies interferentes (I) nos valores de $\mathrm{i}_{\mathrm{p}}$ para uma solução de $\mathrm{As}$ (III) $0,075 \mathrm{mg} \mathrm{L}^{-1}\left(1,0 \mu \mathrm{mol} \mathrm{L}^{-1}\right)$ na presença e ausência de I

\begin{tabular}{cccc}
\hline (I) & $\begin{array}{c}\text { Concentração de I } \\
\left(\mathrm{mg} \mathrm{L}^{-1}\right)\end{array}$ & $\begin{array}{c}\text { Razão } \\
{[\mathrm{As}(\mathrm{III})] /[\mathrm{I}]}\end{array}$ & $\begin{array}{c}\text { Nível de interfe- } \\
\text { rência }(\%)\end{array}$ \\
\hline $\mathrm{Fe}^{3+}$ & 0,15 & 2,7 & +1 \\
$\mathrm{Cu}^{2+}$ & 0,01 & 0,16 & +3 \\
$\mathrm{Al}^{3+}$ & 0,001 & 0,037 & +1 \\
$\mathrm{Zn}^{2+}$ & 0,05 & 0,76 & +3 \\
$\mathrm{~Pb}^{2+}$ & 0,05 & 0,24 & -5 \\
$\mathrm{Ni}^{2+}$ & 0,2 & 3,4 & +3 \\
$\mathrm{Cr}^{3+}$ & 1,0 & 19 & -1 \\
$\mathrm{SO}_{4}{ }^{2-}$ & 0,24 & 2,5 & +4 \\
$\mathrm{PO}_{4}{ }^{3-}$ & 0,35 & 3,7 & +5 \\
$\mathrm{AH}^{2+}$ & 10 & - & +3 \\
$\mathrm{AF}$ & 10 & - & -1 \\
\hline
\end{tabular}

A maior parte dos cátions avaliados apresentou algum grau de interferência no sinal voltamétrico de As(III), causando aumento de $\mathrm{i}_{\mathrm{p}}$. Cobre forma intermetálico com As, podendo diminuir a altura de pico. Por outro lado, o pico de redissolução de $\mathrm{Cu}$ em eletrodo de ouro ocorre em potenciais próximos àquele do As, podendo explicar a interferência positiva. A diminuição de $i_{p}$ na presença de $\mathrm{Pb}(\mathrm{II})$ e $\mathrm{Cr}$ (III) pode estar relacionada com a formação de sais insolúveis destes íons com arsenito. Ânions não apresentaram interferências significativas, assim como ácidos húmico e fúlvico, que são os principais componentes da matéria orgânica de ocorrência natural. 


\section{Aplicação}

Definidas as condições do método, este foi aplicado a amostras de água doce coletadas nos rios Ribeirão do Meio e Lençóis no município de Lençóis, BA, Brasil. Ao se analisar as amostras pelo método de adição de padrão, as concentrações de As(III) obtidas foram 5,7

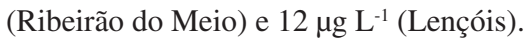

Para avaliar a exatidão do método as amostras de água foram enriquecidas com $10 \mu \mathrm{g} \mathrm{L}^{-1}\left(0,133 \mu \mathrm{mol} \mathrm{L}^{-1}\right)$ de As(III) e analisadas novamente pelo método da adição de padrão, realizando as medidas pelo método SI-SWASV. A porcentagem de recuperação média foi de $108 \pm 12 \%(n=3)$. As mesmas amostras foram analisadas por absorção atômica com atomização eletrotérmica em forno de grafite, obtendo-se uma recuperação média de $95,5 \pm 0,9 \%(n=2)$, indicando que a exatidão do método proposto é aceitável.

\section{CONCLUSÃO}

Foi desenvolvido um método automatizado SI-SWASV para determinação de As(III), obtendo-se limite de quantificação de 5,2 $\mu \mathrm{g} \mathrm{L} \mathrm{L}^{-1}$. Esse limite é quase duas vezes menor do que a concentração máxima de As em águas potáveis permitida pela Organização Mundial de Saúde. A frequência de amostragem foi de 20 determinações por hora empregando instrumentação de baixo custo que pode ser levada a campo. Os sensores impressos de ouro não se mostraram robustos no meio de condicionamento e análise, sendo que a durabilidade dos sensores apresentou grande dependência do lote de fabricação. $\mathrm{O}$ eletrodo de ouro cristalino apresentou repostas reprodutivas em concentrações baixas e altas de $\mathrm{HCl}$, sendo o eletrodo mais adequado para aplicação do método. Assim, a principal contribuição deste trabalho é demonstrar que é possível determinar As(III) nos níveis de concentração máximas permitidas em águas usando instrumentação simples e de baixo custo com eletrodos de ouro disponíveis comercialmente que apresentam um tempo de vida muito maior do que sensores impressos, ou eletrodos de Au obtidos por deposição eletroquímica sobre substratos de carbono. As amostras analisadas apresentaram coloração castanha, sugerindo a presença de concentrações elevadas de matéria orgânica natural, de modo que os resultados de recuperação média ente 95 e $108 \%$ sugerem que o método proposto tem potencial para a aplicação em outras amostras de água naturais.

\section{AGRADECIMENTOS}

FHN agradece à FAPESP (Processo 2012/08065-1) pela bolsa de doutoramento e JCM agradece ao CNPq (Processo 306075/2013-0) pela bolsa de pesquisador. Os autores agradecem ao GAPE, Grupo de Análises e Pesquisa em Espectrometria do Instituto de Química da Universidade de São Paulo, pela realização das análises por absorção atômica.

\section{REFERÊNCIAS}

1. Basu, A.; Saha, D.; Saha, R.; Ghosh, T.; Saha, B.; Res. Chem. Intermed. 2014, 40, 447.

2. Teixeira, M. C.; Tavares, E. D. F. L.; Saczk, A. A.; Okumura, L. L.; Cardoso, M. D. G.; Magriotis, Z. M.; De Oliveira, M. F.; Food Chem. $\mathbf{2 0 1 4}, 154,38$.

3. Hung, D. Q.; Nekrassova, O.; Compton, R. G.; Talanta 2004, 64, 269.

4. Mays, D. E.; Hussam, A.; Anal. Chim. Acta 2009, 646, 6.

5. Ivandini, T. A.; Sato, R.; Makide, Y.; Fujishima, A.; Einaga, Y.; Anal. Chem. 2006, 78, 6291.

6. Grabarczyk, M.; Electroanalysis 2010, 22, 2017.

7. Cavicchioli, A.; La-Scalea, M. A.; Gutz, I. G. R.; Electroanalysis 2004, $16,697$.

8. Ma, J.; Sengupta, M. K.; Yuan, D.; Dasgupta, P. K.; Anal. Chim. Acta 2014, 831,1 .

9. Giacomino, A.; Abollino, O.; Lazzara, M.; Malandrino, M.; Mentasti, E.; Talanta 2011, 83, 1428.

10. Ivaska, A.; Kubiak, W.W.; Talanta 1997, 44, 713.

11. Ribeiro, L. F.; Masini, J. C.; Electroanalysis 2014, 26, 2754

12. Dos Santos, L. B. O.; Silva, M. S. P.; Masini, J. C.; Anal. Chim. Acta 2005, 528, 21.

13. Duangthong, S.; Suwanin, A.; Chooto, P.; Innuphat, C.; ScienceAsia 2016, 42, 266.

14. Punrat, E.; Chuanuwatanakul, S.; Kaneta, T.; Motomizu, S.; Chailapakul, O.; Talanta 2013, 116, 1018.

15. Lolic, A.; Nikolic, S.; Mutic, J.; Anal. Sci. 2008, 24, 877.

16. Sue, J. W.; Ku, H. H.; Chung, H. H.; Zen, J. M.; Electrochem. Commun. 2008, 10, 987.

17. Laschi, S.; Bagni, G.; Palchetti, I.; Mascini, M.; Anal. Lett. 2007, 40, 3002.

18. Laschi, S.; Palchetti, I.; Mascini, M.; Sensors Actuators, B 2006, 114 , 460.

19. Bernalte, E.; Sánchez, C. M.; Gil, E. P.; Anal. Chim. Acta 2011, 689, 60.

20. do Nascimento, F. H.; Masini, J. C.; Anal. Lett. 2016, 49, 699.

21. do Nascimento, F. H.; Masini, J. C.; Talanta 2012, 100, 57.

22. Correia, P. R. M.; Oliveira, P. V.; Talanta 2005, 67, 46.

23. Gibbon-Walsh, K.; Salaün, P.; van den Berg, C. M. G.; Anal. Chim. Acta 2010, 662, 1 . 